\title{
Integrated Photon Upconversion Solar Cell via Molecular Self-Assembled Bilayers
}

\author{
Sean P. Hill, Tristan Dilbeck, Enric Baduell, Kenneth Hanson* \\ Department of Chemistry and Biochemistry, Florida State University, Tallahassee, \\ Florida, 32306, United States
}

\section{Contents}

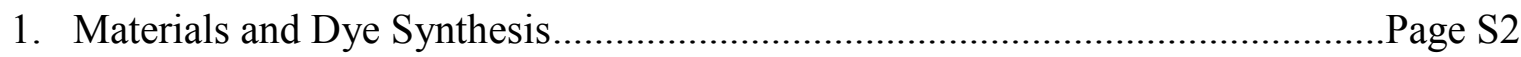

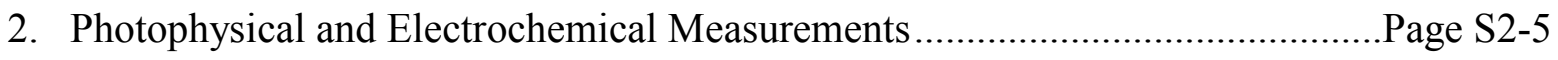

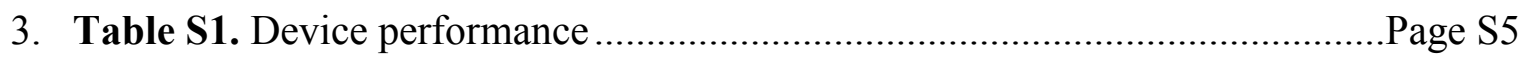

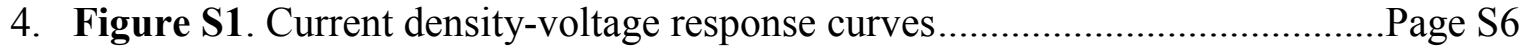

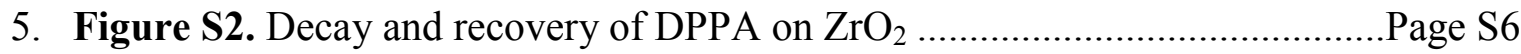

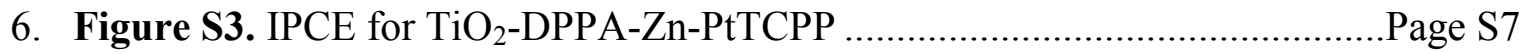

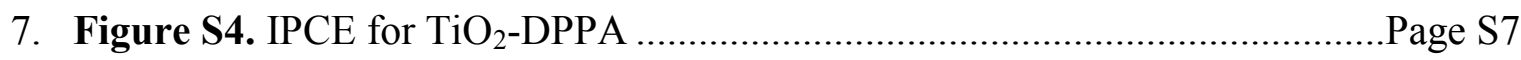

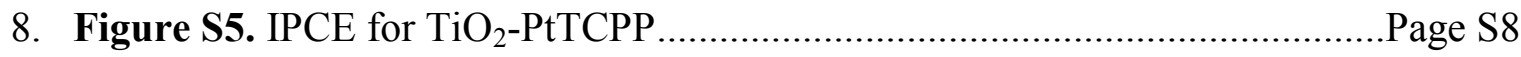

9. Figure S6. Absorption spectra for PtTCPP loading overtime in a bilayer film ..Page S8

10. Figure S7. Emission spectra for $\mathrm{ZrO}_{2}-\mathrm{PtTCPP}$ and bilayer films............................... S9

11. Figure S8. Emission spectra for $\mathrm{ZrO}_{2}-(3)+\mathrm{PtTPP}$ and $\mathrm{ZrO}_{2}-\mathrm{DPPA}+\mathrm{PtTPP}$.......Page S9

12. Figure S9. Emission spectra for bilayer films with and w/out $\left[\mathrm{Co}(\mathrm{bpy})_{3}\right]^{2+/ 3+} \ldots$ Page S10

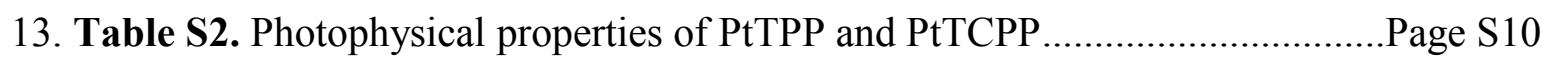

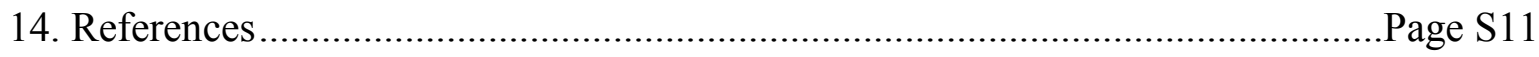




\section{a. Materials and Dye Synthesis}

Materials: Zinc acetate dehydrate, $\mathrm{H}_{2} \mathrm{PtCl}_{6}$, nitrosyl tetrafluoroborate, cobalt (II) chloride, ammonium hexafluorophosphate (Sigma-Aldrich), 2,2‘ bipyridine (Oxchem), Pt(II) meso-tetraphenylporphine, and $\mathrm{Pt}(\mathrm{II})$ meso-tetra(4-carboxyphenyl)porphine (Frontier Scientific), were purchased from their respective suppliers, in parentheses, and used as received. All other reagents and solvents (analytical reagent grade) have been purchased and used without further purification from Alfa Aesar. Fluorine-doped tin oxide (FTO) coated glass (sheet resistance $15 \Omega \square^{-1}$ ) was purchased from Hartford Glass Co. Meltonix film (1170-25), Ti-Nanoxide T solgel paste (11421) and Vac'n Fill Syringe (65209) were purchased from Solaronix. Micro glass cover slides $(18 \times 18 \mathrm{~mm})$ were obtained from VWR. $\mathrm{ZrO}_{2}$ solgel paste was prepared following a previously reported procedure. ${ }^{1}$

Dye Synthesis: 4,4'-(Anthracene-9,10-diyl)bis(4,1-phenylene) diphosphonic acid $(\mathrm{DPPA})^{2}$ and Tris-(2,2'-bipyridine)cobalt(II) di(hexafluorophosphate) $\left(\mathrm{Co}(\mathrm{bpy})_{3}\left(\mathrm{PF}_{6}\right)_{2}\right)^{3}$ were prepared by following previously published procedures.

\section{b. Photophysical and Electrochemical Measurements}

Energy Transfer Measurements: The efficiency of PtTPP or PtTCPP to DPPA energy transfer for $\mathrm{ZrO}_{2}$-DPPA-Zn-PtTCPP and $\mathrm{ZrO}_{2}$-DPPA with PtTPP in solution, respectively, was quantified using steady state emission experiments. The sensitizer was excited $510 \mathrm{~nm}$ light from a Xe lamp and emission was measured from 600-800 nm. Phosphorescence quenching was calculated using equation S1 where $\mathrm{I}_{\mathrm{SA}}$ is the integrated emission intensity from $600-800 \mathrm{~nm}$ for $\mathrm{ZrO}_{2}$-DPPA-Zn-PtTCPP or $\mathrm{ZrO}_{2}$-DPPA with 
PtTPP in solution, and $\mathrm{I}_{\mathrm{S}}$ is the emission intensity for $\mathrm{ZrO}_{2}-\mathrm{PtTCPP}$ or $\mathrm{ZrO}_{2}-(3)$ with PtTPP in solution.

$$
\% \text { Quenching }=\left(1-\frac{\sum I_{S A}}{\sum I_{S}}\right) \times 100
$$

For the heterogeneous PtTPP only sample, the surface of $\mathrm{ZrO}_{2}$ was functionalized with P, P'-Terphenyl-4,4"-bis-phosphonic acid (3) which is a photo- and electrochemically inert surrogate for DPPA. ${ }^{4}$ The addition of (3) was found to be necessary to prevent PtTPP aggregation on the surface of $\mathrm{ZrO}_{2}$, which lead to quenching of the phosphorescence in the $\mathrm{ZrO}_{2}$ with PtTPP samples.

Incident Photon to Current Efficiency: IPCE was acquired every $5 \mathrm{~nm}$ (5 nm bandwidth) with excitation intensities measured at each wavelength $\left(I_{e x}\right)$ to correct for variation throughout the spectrum. \% IPCE was calculated using Equation S2.

$$
\% I P C E=\frac{\left(J_{s c}\right)(1240)}{\left(I_{e x}\right)(\lambda)}
$$

Absorption Spectra: Data were recorded on an Agilent 8453 UV-visible photo diode array spectrophotometer. Thin film absorption spectra were obtained by placing dry, derivatized $\mathrm{TiO}_{2}$ and $\mathrm{ZrO}_{2}$ slides perpendicular to the detection beam path.

Steady-State Emission: Data were collected at room temperature using an Edinburgh FLS980 fluorescence spectrometer. The samples were excited using the output from either a housed $450 \mathrm{~W}$ Xe lamp/single grating $\left(1800 \lambda \mathrm{mm}^{-1}, 250 \mathrm{~nm}\right.$ blaze $)$ Czerny-Turner monochromator or a Nd:YAG laser (Aixiz, AD-532-400T). The output from the Nd:YAG laser was passed through a variable neutral density filter (Edinburgh 
F-B01 laser mount), a $2 \mathrm{~mm}$ diameter iris (Newport ID-1.0) and then directed to the sample via a flip mirror. Emission from the sample was first passed through a long pass filter or a $532 \mathrm{~nm}$ notch filter (Thorlabs Inc., NF533-17), then a single grating (1800 $\lambda$ $\mathrm{mm}^{-1}, 500 \mathrm{~nm}$ blaze) Czerny-Turner monochromator and finally detected by a Peltiercooled Hamamatsu R928 photomultiplier tube. Laser intensities were measured using a power meter (Ophir Vega, 7Z01560) with a high sensitivity power sensor (Ophir, 3A-FS 7Z02628).

Time-Resolved Emission: The excited state lifetimes for PtTCPP in the bilayer on $\mathrm{ZrO}_{2}$ and PtTPP in the presence of $\mathrm{ZrO}_{2}$-DPPA were acquired using the FLS980's multichannel scaling (MCS) acquisition mode with $510 \mathrm{~nm}$ excitation from a $60 \mathrm{~W}$ microsecond flashlamp (pulse width $<2.5 \mu \mathrm{s}$ ) at a $100 \mathrm{~Hz}$ repetition rate. Emission was passed through a $570 \mathrm{~nm}$ long pass filter placed in front of the detector. Decay traces were fit using the Edinburgh software package.

Amperometric $i-t$. Data were collected using a $\mathrm{CH}$ Instruments CHI630E electrochemical analyzer using a two-electrode configuration $\left(\mathrm{TiO}_{2}\right.$ working, $\mathrm{Pt}$ counter $)$ held at $0 \mathrm{~V}$ applied potential. The samples were irradiated with either an AM1.5 solar simulator (Light Model 66181 oriel corrected with a standard air-mass filter) passing through a $375 \mathrm{~nm}$ or $495 \mathrm{~nm}$ long pass filters, with $532 \mathrm{~nm}$ from a Nd:YAG laser (Aixiz, AD-532-400T) or with $366 \mathrm{~nm}$ LED (ThorLabs M365L2-UV) used as the light source. The intensity of solar irradiance was controlled by varying the distance between the solar simulator source and sample. The laser light intensity was controlled using a neutral 
density filter as described above for the photophysical measurements. A Model T132 Shutter Driver/Timer (UniBlitz) coupled to a mechanical shutter (Vincent Associates, VS25) was placed between the light source and sample to control $10 \mathrm{~s}$ light-dark intervals over a 70 s time period.

Table S1. Performance characteristics of DSSCs containing monolayer and bilayer photoanodes under AM1.5 irradiation with $375 \mathrm{~nm}$ and $495 \mathrm{~nm}$ long pass filters.

\begin{tabular}{lcccc} 
& Filter $[\mathrm{nm}]$ & $\begin{array}{c}J_{\mathrm{sc}} \\
{\left[\mu \mathrm{A} / \mathrm{cm}^{2}\right]}\end{array}$ & $\begin{array}{c}V_{\mathrm{oc}} \\
{[\mathrm{mV}]}\end{array}$ & \multicolumn{1}{c}{$\eta$} \\
DPPA & 375 & 114.6 & 22.5 & $6.3 \times 10^{-6}$ \\
\hline PtTCPP & 375 & 21.9 & 6.0 & $3.2 \times 10^{-7}$ \\
\hline Bilayer & 375 & 204.0 & 115.0 & $6.8 \times 10^{-5}$ \\
\hline DPPA & 495 & 2.9 & 0.5 & $3.9 \times 10^{-9}$ \\
\hline PtTCPP & 495 & 3.6 & 1.0 & $8.6 \times 10^{-9}$ \\
\hline Bilayer & 495 & 9.0 & 7.2 & $1.6 \times 10^{-7}$ \\
\hline & & & & \\
\hline
\end{tabular}




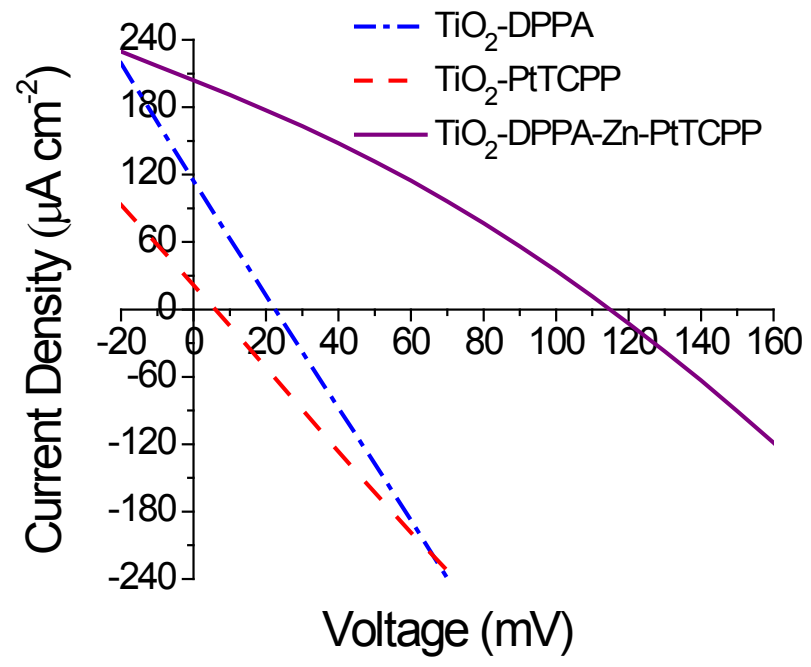

Figure S1. Current density-voltage response curves for $\mathrm{TiO}_{2}-\mathrm{DPPA}, \mathrm{TiO}_{2}-\mathrm{PtTCPP}$, and $\mathrm{TiO}_{2}$-DPPA-Zn-PtTCPP with $\left[\mathrm{Co}(\mathrm{bpy})_{3}\right]^{2+/ 3+}$ inMeCN under 1 sun (AM1.5) illumination with a $375 \mathrm{~nm}$ long pass filter.

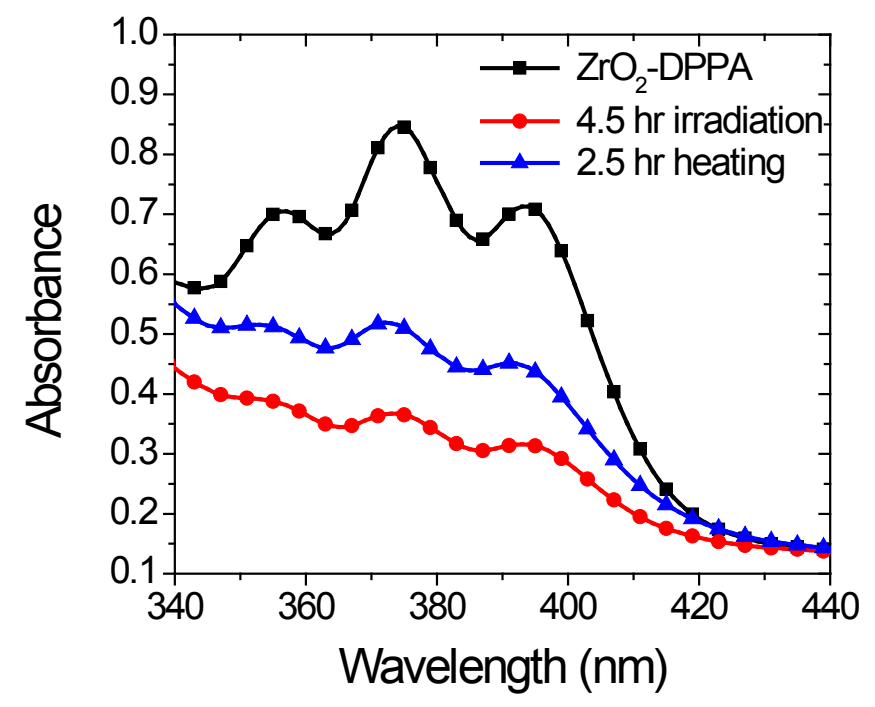

Figure S2. Absorbance spectra for DPPA loaded onto $\mathrm{ZrO}_{2}$ (black, square) after irradiation under white light for $4.5 \mathrm{hr}$ (red, circle) followed by $2.5 \mathrm{hr}$ heating at $70{ }^{\circ} \mathrm{C}$ (blue, triangle). 


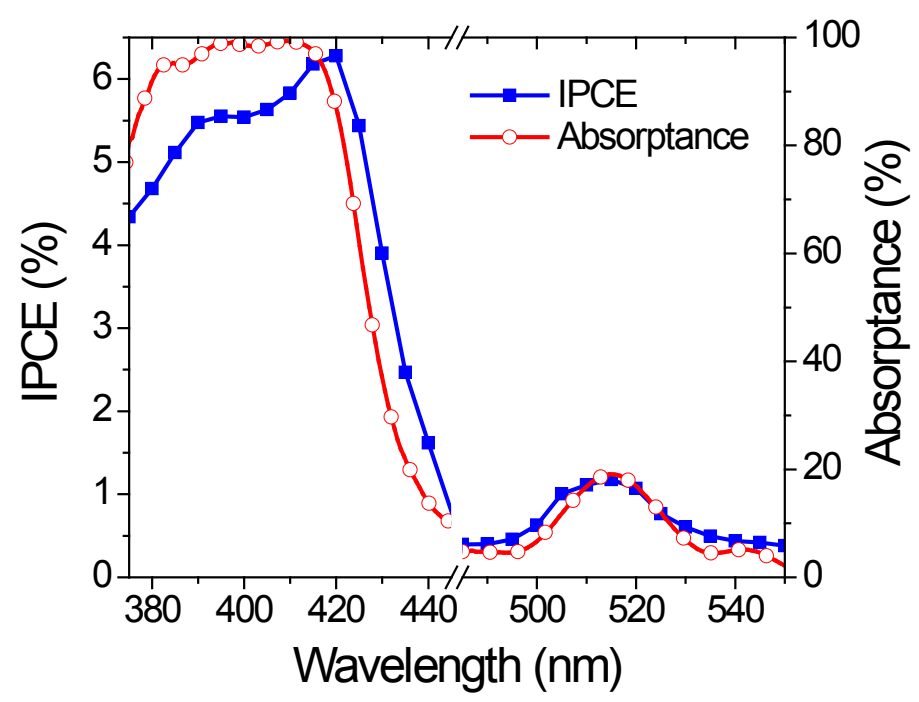

Figure S3. IPCE (blue, square) and absorptance (red, circle) for $\mathrm{TiO}_{2}$-DPPA-Zn-PtTCPP with $\left[\mathrm{Co}(\mathrm{bpy})_{3}\right]^{2+/ 3+}$ in $\mathrm{MeCN}$.

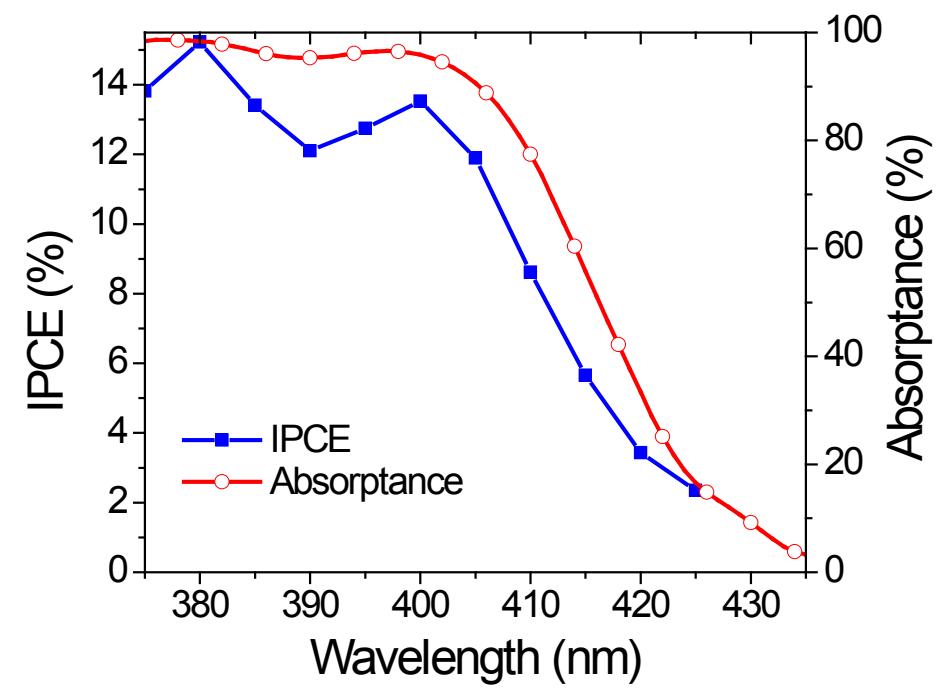

Figure S4. IPCE (blue, square) and absorptance (red, circle) for $\mathrm{TiO}_{2}$-DPPA with $\left[\mathrm{Co}(\mathrm{bpy})_{3}\right]^{2+/ 3+}$ in $\mathrm{MeCN}$. 


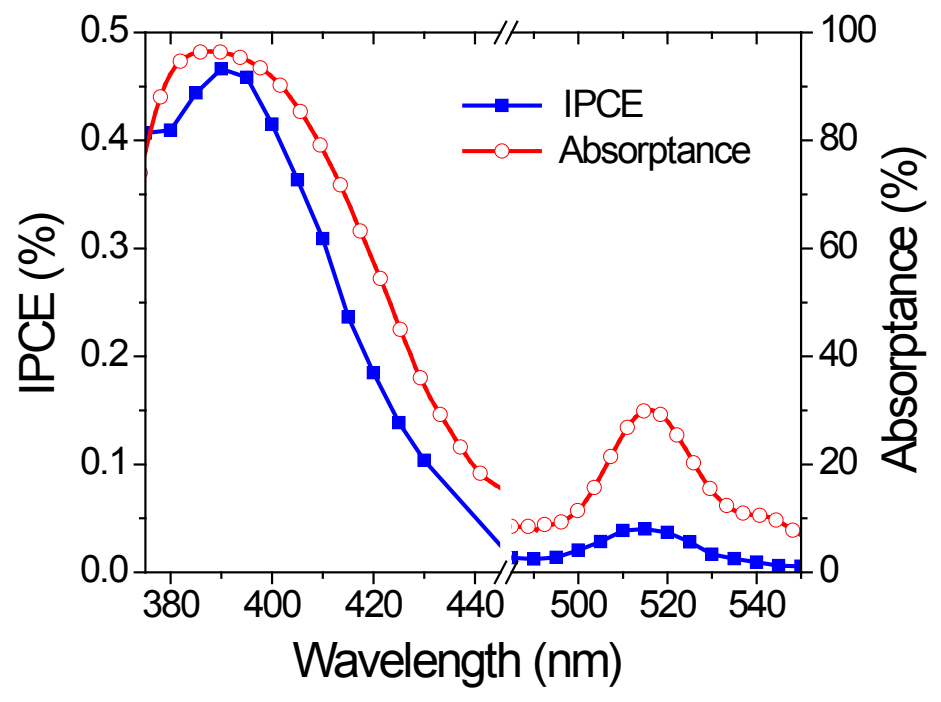

Figure S5. IPCE (blue, square) and absorptance (red, circle) for $\mathrm{TiO}_{2}-\mathrm{PtTCPP}$ with $\left[\mathrm{Co}(\text { bpy })_{3}\right]^{2+/ 3+}$ in $\mathrm{MeCN}$.

a)

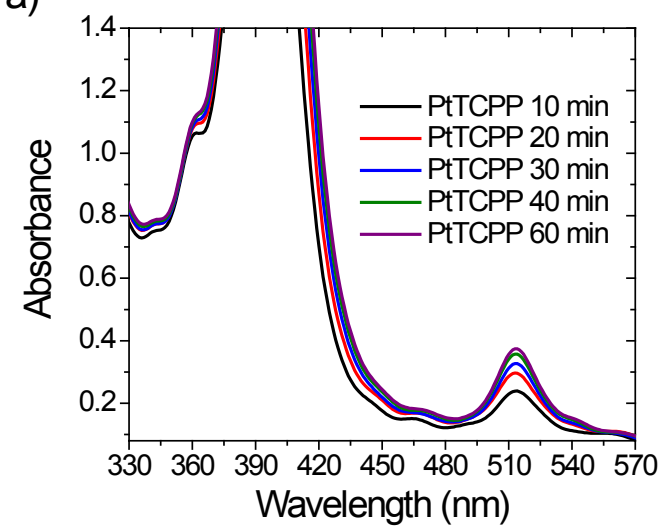

b)

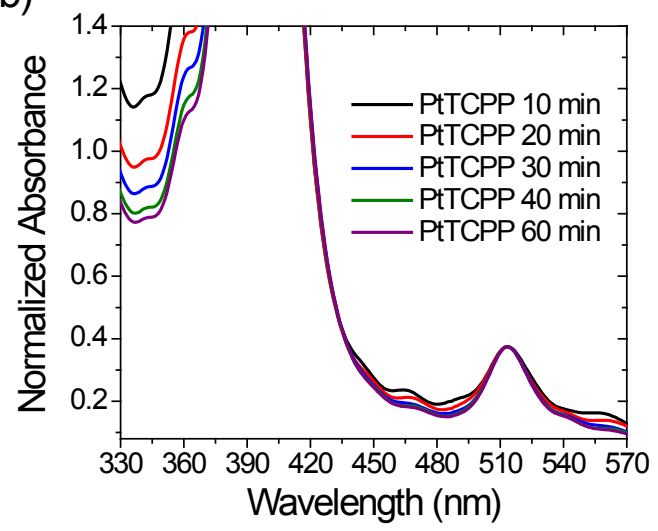

Figure S6. Absorption spectra of $\mathrm{ZrO}_{2}$-DPPA-Zn-PtTCPP after soaking in $200 \mu \mathrm{M}$ PtTCPP in DMSO for 10, 20 30, 40 and 60 minutes (a) as recorded and (b) normalized at $512 \mathrm{~nm}$. 


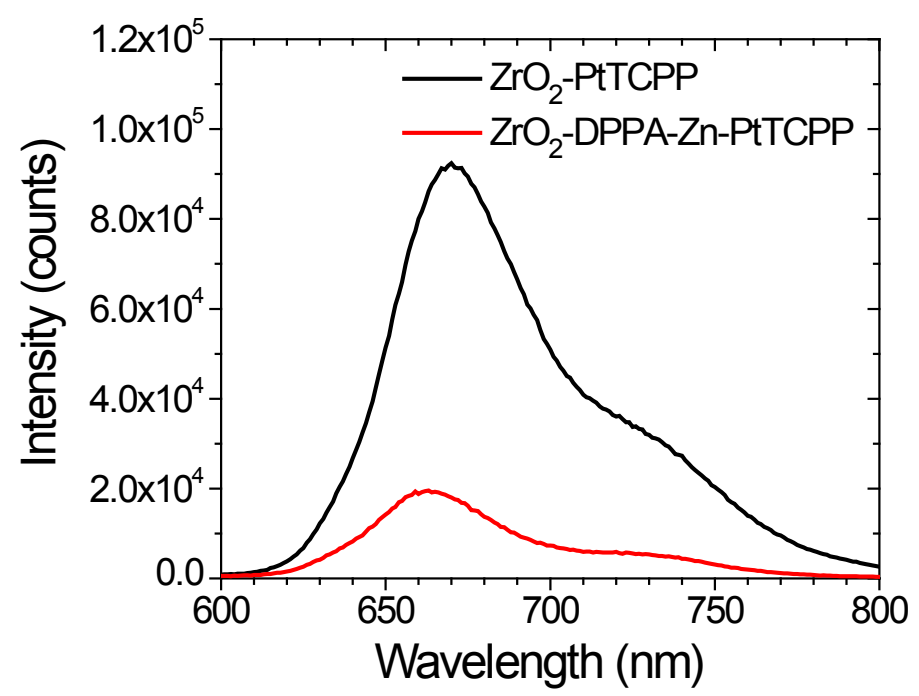

Figure S7. Emission from $\mathrm{ZrO}_{2}$-PtTCPP (black) and $\mathrm{ZrO}_{2}$-DPPA-Zn-PtTCPP (red) in DMF under $510 \mathrm{~nm}$ excitation (570 nm long pass filter).

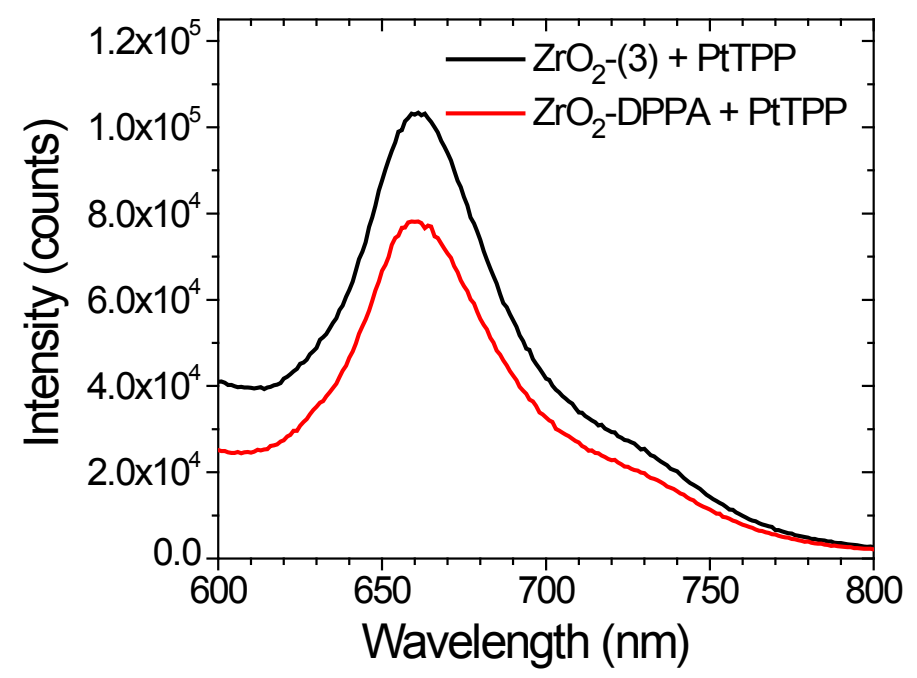

Figure S8. Emission from $\mathrm{ZrO}_{2}-(3)+$ PtTPP (black) and $\mathrm{ZrO}_{2}-\mathrm{DPPA}+$ PtTPP (red) in DMF under $510 \mathrm{~nm}$ excitation (570 $\mathrm{nm}$ long pass filter). 


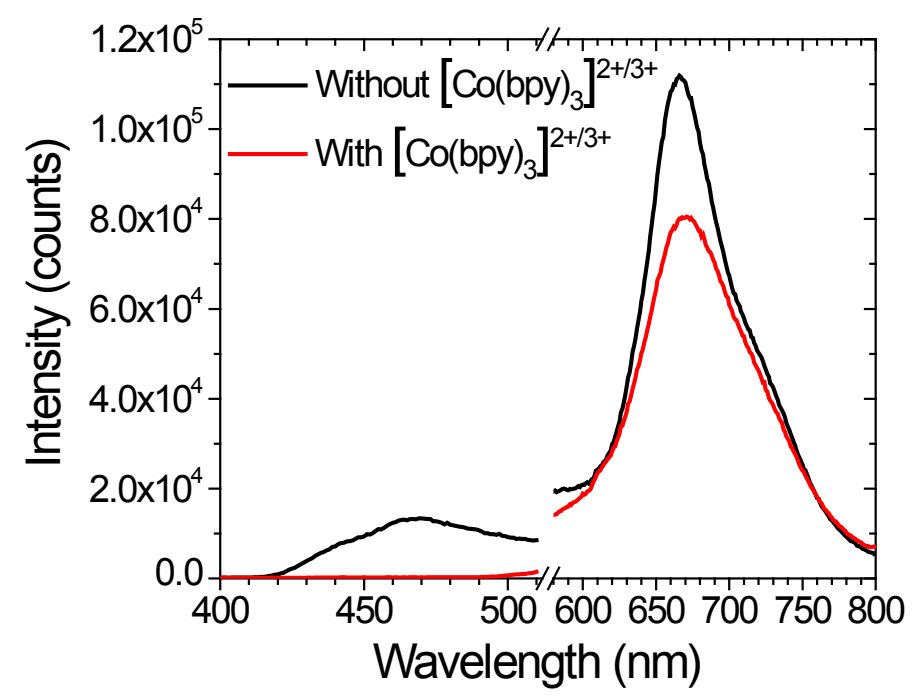

Figure S9. Emission from $\mathrm{ZrO}_{2}$-DPPA-Zn-PtTCPP without (black) and with (red) $\left[\mathrm{Co}(\mathrm{bpy})_{3}\right]^{2+/ 3+}$ in $\mathrm{MeCN}$ under $532 \mathrm{~nm}$ laser excitation (532 nm notch filter).

Table S2. Photophysical properties of PtTPP and PtTCPP.

\begin{tabular}{|c|c|c|c|c|c|c|c|c|c|}
\hline & $\begin{aligned} & \lambda_{\mathrm{abs}}{ }^{a}[\mathrm{~nm}][\varepsilon, \\
\times & \left.10^{4} \mathrm{M}^{-1} \mathrm{~cm}^{-1}\right]\end{aligned}$ & $\begin{array}{c}\lambda_{\mathrm{em}} \\
{[\mathrm{nm}]}\end{array}$ & $\begin{array}{c}\tau^{b} \\
{[\mu \mathrm{s}]}\end{array}$ & $\Phi$ & $\begin{array}{c}k_{\mathrm{r}}^{c} \\
{\left[\times 10^{2} \mathrm{~s}^{-1}\right]}\end{array}$ & $\begin{array}{c}k_{\mathrm{nr}}{ }^{d} \\
{\left[\times 10^{4} \mathrm{~s}^{-1}\right]}\end{array}$ & $\begin{array}{l}E_{1 / 2}{ }^{\mathrm{ox}} \\
{[\mathrm{V} \text { vs }} \\
\text { NHE] }\end{array}$ & $E_{1 / 2}\left(\mathrm{~S}_{1}\right)^{\mathrm{e}}$ & $E_{1 / 2}\left(\mathrm{~T}_{1}\right)^{\mathrm{f}}$ \\
\hline PtTPP & $\begin{array}{c}401(27.46) \\
509(2.72), 539 \\
(0.73)\end{array}$ & 666 & 43 & 0.014 & 3.26 & 2.29 & 1.23 & -1.02 & -0.63 \\
\hline PtTCPP & $\begin{array}{c}403(27.55) \\
510(2.90), 539 \\
(0.77)\end{array}$ & 666 & 53 & 0.018 & 3.40 & 1.85 & 1.25 & -0.99 & -0.61 \\
\hline
\end{tabular}

\footnotetext{
${ }^{a}$ Measured in a DMF solution at each peak maxima. ${ }^{b}$ Lifetime from an exponential tail fit to the excited state decay (ex: $510 \mathrm{~nm}) \cdot{ }^{c} k_{\mathrm{r}}=\Phi / \tau \cdot{ }^{d} k_{\mathrm{nr}}=(1-\Phi) / \tau .{ }^{\mathrm{e}}$ The singlet excited state reduction potential. ${ }^{\mathrm{f}}$ The triplet excited state reduction potential.
} 


\section{References:}

(1) Heimer, T. A.; DArcangelis, S. T.; Farzad, F.; Stipkala, J. M.; Meyer, G. J. An Acetylacetonate-Based Semiconductor-Sensitizer Linkage. Inorg. Chem. 1996, 35, 5319.

(2) Hill, S. P.; Banerjee, T.; Dilbeck, T.; Hanson, K. Photon Upconversion and Photocurrent Generation via Self-Assembly at Organic-Inorganic Interfaces. $J$. Phys. Chem. Lett. 2015, 6, 4510.

(3) Feldt, S. M.; Gibson, E. A.; Gabrielsson, E.; Sun, L.; Boschloo, G.; Hagfeldt, A. Design of Organic Dyes and Cobalt Polypyridine Redox Mediators for HighEfficiency Dye-Sensitized Solar Cells. J. Am. Chem. Soc. 2010, 132, 16714.

(4) Wang, J. C.; Murphy, I. A.; Hanson, K. Modulating Electron Transfer Dynamics at Dye-Semiconductor Interfaces via Self-Assembled Bilayers. J. Phys. Chem. C 2015, 119,3502 . 